\title{
Selective transmission of multidrug resistant HIV to a newborn related to poor maternal adherence
}

\author{
N Desai, M Mathur
}

Sex Transm Infect 2003;79:419-421

\begin{abstract}
Objectives: To report perinatal transmission of multidrug resistant (MDR) HIV related to variable maternal adherence antenatally.

Methods: Case study including review of clinic records, adherence information, laboratory data, and HIV genotyping results in mother and infant.

Results: Poor maternal adherence to clinic visits and antiretroviral therapy contributed to detectable viraemia antenatally. When tested for the first time at age 6 months, the infant was found to have virus with resistance to multiple drugs. In this case, prophylaxis with zidovudine (AZT) failed to prevent the transmission of the MDR strain.

Conclusions: Perinatal transmission of MDR HIV can occur despite standard peripartum prophylaxis with AZT. Perinatal prophylaxis should be tailored to the mother's treatment history and resistance profile. Paediatric HIV specialists should be prepared to deal with a small, but slowly increasing number of babies with a "nightmare" multidrug resistant virus with limited treatment options.
\end{abstract}

$\mathrm{T}$ he current recommendations for perinatal prophylaxis with zidovudine (AZT) have resulted in a significant reduction of perinatal transmission of wild type HIV. However, there is a $9-17 \%$ prevalence of AZT resistance in tested perinatally infected infants in various US cohorts. AZT resistance has been associated in some studies with an increased transmission risk. ${ }^{1-4}$

In a recent women and infant transmission study including 1542 HIV infected women with singleton live births, factors affecting perinatal transmission included levels of HIV-RNA (viral load) at delivery. This risk increases 2.4-fold for every $\log 10$ increase in viral load (VL), with effective highly active antiretroviral therapy (HAART) being associated with a low $1.2 \%$ rate of transmission. ${ }^{5}$ The emergence of drug resistance may be a barrier to further reduction in perinatal HIV transmission rates.

We describe a baby with a multidrug resistant (MDR) HIV born to a mother variably adherent to HAART antenatally. Transmission occurred despite standard peripartum prophylaxis with AZT. When tested for the first time at age 6 months, the infant was found to have virus with detectable resistance to multiple drugs.

\section{METHODS}

Review of clinic records, adherence information, laboratory data, and HIV genotyping results in mother and infant revealed the following information.

\section{Mother}

The mother was known to be HIV positive for 5 years before pregnancy. The only previous antiretroviral therapy (ART) was AZT/lamivudine (3TC)/nevirapine (NVP). Adherence was assessed by documentation of missed clinic appointments, direct questioning regarding missed doses, and pharmacy feedback on timing of refills. She was poorly adherent in all respects.

\section{Antenatal course}

This 19 year old primigravida stopped all medications when she became pregnant and first sought care at 20 weeks gestation. Viral load (VL; copies/ml) (Roche Amplicor Monitor version 1.5 (quantitative RNA PCR), Roche Molecular systems, Somerville, NJ, USA, limits of detection: 400 000-750 000 copies $/ \mathrm{ml}$ ) was 11900 . Resistance testing was not done at this time since the patient had stopped taking her prescribed medications for about 4 months. She agreed to restart HAART, but despite medical advice recommending a protease inhibitor (PI) based regimen, would only accept her previous regimen (AZT/3TC/ NVP). She was again intermittently adherent, but better than before. She had a total of seven antenatal clinic visits. VL was $<400$ at 24 weeks, 459 at 32 weeks, and 968 copies/ml at 34 weeks gestation.

\section{Perinatal course}

The mother refused elective caesarean section. Labour was induced using an escalating oxytocin infusion at 42 weeks gestation. After 16 hours with minimal uterine contractions, labour was augmented by artificial rupture of amniotic membranes (ARM). The baby was born by vaginal delivery with vacuum extraction assistance and a midline episiotomy 45 minutes after ARM. No internal fetal monitors were used and there was no chorioamnionitis. Mother and baby received standard AZT prophylaxis. The mother refused combination antiretroviral prophylaxis for the baby. The baby was exclusively formula fed.

\section{Postpartum course}

The mother insisted on continuing AZT/3TC/NVP, contrary to medical advice recommending a PI based combination. Even using her preferred combination, poor adherence was ongoing. Repeated recommendations were made to change but she agreed only to daily efavirenz (EFV) with AZT and 3TC 2 years post partum. She made this decision despite counselling regarding the high likelihood of resistance and limited efficacy of this regimen.

\section{Newborn}

The baby was diagnosed with HIV infection by ELISA and HIV DNA PCR positive $\times 2$ (Wadsworth laboratory, NYS Department of Health, Albany, NY). The virus was Clade B.

\section{Resistance testing}

HIV genotyping (Virologic Inc, South San Francisco, CA, USA) was performed on the baby at age 6 months. The only antiretroviral agent the baby had received was postpartum AZT for the first 6 weeks of life. Genotyping was repeated on 


\begin{tabular}{|c|c|c|c|c|}
\hline & Viral load & $\begin{array}{l}\text { Treatment regimen } \\
\text { at testing }\end{array}$ & Reverse transcriptase mutations & Protease mutations \\
\hline $\begin{array}{l}\text { Baby age } 6 \\
\text { months }\end{array}$ & 443606 & None & $\begin{array}{l}\text { Primary: K103N (all NNRTI) } \\
\text { M184V (3TC, FTC, ddC, abacavir) } \\
\text { secondary: E63E, R21 1K, L214F }\end{array}$ & $\begin{array}{l}\text { Primary: L33V odd (RTV) } \\
\text { L63V odd (IDV, NFV) } \\
\text { secondary: } 113 \mathrm{~V}, \mathrm{~N} 37 \mathrm{C} \text {, } \\
164 \mathrm{~V}, 172 \mathrm{~V}\end{array}$ \\
\hline $\begin{array}{l}\text { Mother } 7 \\
\text { months post } \\
\text { partum }\end{array}$ & 10756 & None & $\begin{array}{l}\text { Primary: None Secondary: R211K, } \\
\text { L214F }\end{array}$ & $\begin{array}{l}\text { Primary: } L 33 \mathrm{~V} \text { L63V } \\
\text { secondary: } 113 \mathrm{~V}, \mathrm{~N} 37 \mathrm{C} \text {, } \\
\text { 164V, E65D, I72V }\end{array}$ \\
\hline $\begin{array}{l}\text { Mother } 2 \frac{1}{2} \\
\text { years post } \\
\text { partum }\end{array}$ & 33670 & $\begin{array}{l}\text { AZT/3TC/efavirenz } \\
\text { inconsistent adherence }\end{array}$ & $\begin{array}{l}\text { Primary: K103N (all NNRTI) } \\
\text { secondary: K2OR, V35V, V60V, } \\
\text { Q102K, K122E, Q278H, C162S, } \\
\text { R211K, R277K, E297E }\end{array}$ & $\begin{array}{l}\text { Primary: } L 33 \mathrm{~V} L 63 \mathrm{~V} \\
\text { secondary: } 113 \mathrm{~V}, \mathrm{~N} 37 \mathrm{C} \text {, } \\
163 \mathrm{~V}, 164 \mathrm{~V}, \mathrm{E} 65 \mathrm{D}, 172 \mathrm{~V}\end{array}$ \\
\hline
\end{tabular}

the mother's virus at 7 months and $2 \frac{1}{2}$ years post partum (table 1).

\section{Infant's clinical course}

Based on genotyping results at 6 months of age, the baby was started on antiretroviral therapy with stavudine (D4T), didanosine (ddI), and nelfinavir. He demonstrated a $>2 \log$ fall from a viral load of $>5 \operatorname{logs}$ baseline and continues to do well at age 36 months. VL has been continuously $<400$ copies/ml for over 2 years and latest CD4 count is $1226(30 \%)$.

\section{DISCUSSION}

The baby was born infected with a virus resistant to 3TC and NVP; however, genotyping on the mother 7 months post partum revealed a strain that remained sensitive. In this case, a MDR virus was transmitted to the newborn, but maternal genotyping results post partum do not show the same degree of resistance. The likely explanation for these findings is that the inconsistent maternal adherence to the AZT/3TC/NVP regimen had led to the emergence of a resistant strain in the mother ante partum, and it was this strain that was transmitted to the infant. It is possible that when the first genotyping was performed in the mother at 7 months post partum off therapy a wild type strain had re-emerged with no detection of the multidrug resistant strain that was passed on to the baby. However repeat genotyping after another $2 \frac{1}{2}$ years of inconsistent therapy in the mother showed emergence of resistance to NNRTIs. The resistant strain persisted in the absence of drug pressure, and re-emerged after poor adherence to reinstituted therapy.

This mother had poor adherence to prescribed medications as a result of various concerns. Apprehension about medication related side effects and harming the fetus persisted despite ongoing education, pharmacy, nursing and case manager support, psychosocial support, and medical reassurances. This may be consistent with the psychological and emotional make up of a young first time mother. The team caring for her actively attempted to engage her by intensive health and adherence related education. The psychologist on the team was made available for counselling. The pharmacy was alerted to anticipate poor adherence. Alarm beepers and other adherence promoting measures such as pillboxes and repeated telephone follow up were also instituted. Despite all these measures, she agreed only to use the regimen that she had previous experience with, and that with poor adherence.

In this case, poor maternal adherence contributed to the transmission of a multidrug resistant virus to the newborn, even though it appears that the wild type virus from the mother would have been suppressed by the standard prophylaxis with AZT that was used. Interestingly, the transmission occurred despite the maternal plasma VL being $<1000$ copies/ml in late pregnancy (34 weeks). In view of her poor adherence, the VL may have been higher in the plasma by the time she delivered at 42 weeks gestation and higher still in the genital tract. She was counselled about elective caesarean section as a means of reducing transmission, but refused. Though the duration of rupture of membranes was quite short, and there were no features of chorioamnionitis, her choice of vaginal delivery and the process of active labour may have contributed to transmission.

This report highlights that perinatal transmission does occur despite best efforts using current recommendations. Poor maternal adherence led to the emergence of drug resistance and the baby being infected with a multidrug resistant virus. The likelihood of perinatal infection with resistant HIV will depend on maternal viral load, previous maternal treatment, viral fitness of the resistant strain in the mother, and the mode and timing of delivery.

Suppression of maternal viral load with effective antenatal HAART together with individually tailored perinatal prophylaxis based on maternal treatment history and resistance profile coupled with the optimal mode of delivery is critical in minimising perinatal transmission. It will enable paediatric HIV specialists to prevent perinatal transmission of MDR HIV. Close and intensive collaboration between the healthcare teams managing the mother-infant pair through pregnancy, delivery and post partum is important in achieving this goal. At our institution, a perinatal collaborative team (with adult, adolescent, and paediatric HIV providers, OB-GYN and neonatology) discusses and tracks all HIV positive pregnancies and individualises perinatal prophylaxis based on resistance testing in late pregnancy.

Paediatric HIV specialists should be prepared for managing infants infected with multidrug resistant virus and limited treatment options.

\section{CONTRIBUTORS}

ND followed the child in his clinic and collected the clinical details; MM reviewed the maternal clinic and hospital charts. Both authors participated in the preparation of the manuscript.

\section{Authors' affiliations}

N Desai, M Mathur, Children's Hospital at SUNY Downstate Medical Center, Brooklyn, NY, and The Brooklyn Pediatric AIDS Network, Brooklyn, USA

There are no conflicts of interest or financial disclosures applicable. Presented, in part, at the 40th annual meeting of the IDSA, October 2002, Chicago, IL, USA. 
Correspondence to: Ninad Desai, MD, 450 Clarkson Avenue, Box 1241, Brooklyn, NY 11203, USA; bpandesai@aol.com

Accepted for publication 29 April 2003

\section{REFERENCES}

1 Welles S, Pitt J, Colgrove R, et al. HIV-1 genotypic zidovudine drug resistance and the risk of maternal-infant transmission in the Women and Infants Transmission Study. AIDS 2000;14:263-71.
2 Palumbo $\mathbf{P}, \mathrm{Holl}$ and $\mathrm{B}$, Dobbs $\mathrm{T}$, et al. Antiretroviral resistance mutations among pregnant HIV-1-infected women and their newborns in the US: vertical transmission and clades. J Infect Dis 2001;184:1120-6.

3 Fiscus S, Adimora A, Schoenbach $\mathrm{V}$, et al. Trends in HIV counseling, testing and antiretroviral treatment of HIV-infected women and perinatal transmission in North Carolina. J infect Dis 1999; 180:99-105.

4 Johnson V, Petropoulos C, Woods C, et al. Vertical transmission of multi-drug resistant HIV-1 and continued evolution of drug resistance in an HIV-1 infected infant. J Infect Dis 2001;183:1688-93.

5 Cooper E, Charurat M, Mofenson L, et al. Combination antiretroviral strategies for the treatment of pregnant HIV-1-infected women and prevention of perinatal HIV-1 transmission. J Acquir Immune Defic Syndr 2002;29:484-94.

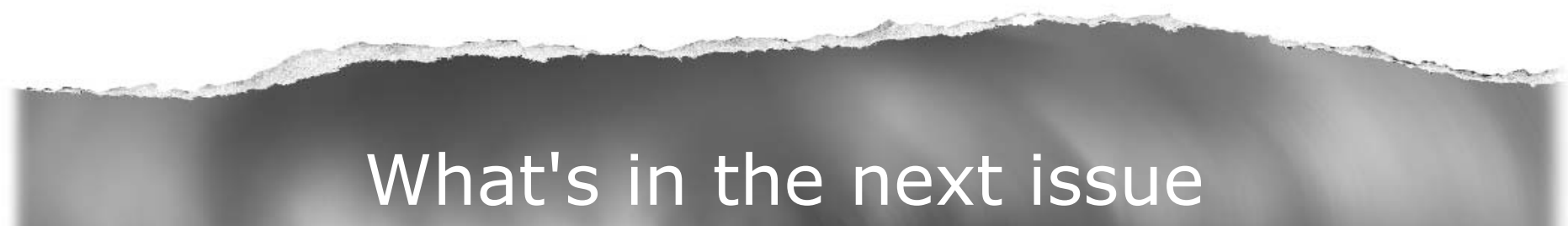

\section{Future content}

See which articles have just been accepted for publication and preview the table of contents for the next issue a month before it is published

\section{www.stijournal.com}

